\title{
Leiffaden für die röntgenologische Beurteilung bei der Kaufuntersuchung des Pferdes (Röntgenleitfaden)
}

\begin{abstract}
Vorwort
Die röntgenologische Untersuchung von Pferden zum Zwecke des Kaufs ist weltweit eine übliche und etablierte Methode. Die Röntgenaufnahmen als bleibende Dokumente werden in der Regel von mehreren Fachkollegen beurteilt und interpretiert. Es ist deshalb nicht selten, dass dabei unterschiedliche Meinungen geäußert werden. Die Differenz der Meinungen war dann stets der Nährboden für gerichtlich ausgetragene Streitigkeiten. Diese Problematik ist bereits im Jahre 1991 vom Ausschuss "Pferde" der Deutschen Tierärzteschaft aufgegriffen worden. Die Lösung sah man darin, eine Kommission zu gründen, die Richtlinien zu den Qualitätsansprüchen der Standardaufnahmen, zur Nomenklatur der röntgenologischen Befunde und Hinweise auf die Interpretation der röntgenologischen Veränderungen erstellt. Die Kommission bestand aus den Professoren Ueltschi aus Bern, Dik aus Utrecht und Hertsch aus Hannover. Der Vorsitz wurde dem Unterzeichnenden vom Ausschuss übertragen. Das Ergebnisprotokoll der Treffen der Röntgenkommission wurde im Jahre 1993 dem Ausschuss "Pferde" vorgestellt.

Es enthielt folgende Abschnitte: 1. Anmerkungen zu Qualität und Technik; 2. Empfehlungen zur Technik; 3. Befundbeschreibung; 4. Befundbeurteilung; 5. Die Einteilung in Röntgenklassen; 6. Röntgenskizzen.

Nach Prüfung und Genehmigung durch verschiedene Fachgremien hat der Ausschuss "Pferde" der Deutschen Tierärzteschaft den Einsatz dieses Röntgenprotokolls für die Beurteilung von Röntgenaufnahmen in der Standardprojektion beim Kauf von Pferden empfohlen. Im Laufe der Jahre hat sich dieses Protokoll als vorteilhaft erwiesen. Die Vorteile dieser Interpretationshilfe hatten sich bald herausgestellt. Die einheitliche Vorgehensweise und Beurteilung half allen Beteiligten und schützte die Tierärzte vor unangebrachten Erwartungen und Forderungen. Ihre neutrale Position wurde gefestigt. Die Einteilung in Klassen machte dem Laien das Ergebnis besser verständlich. Natürlich gab es immer wieder Interessengruppen, die sich gegen eine derartige Einteilung strüubten, insbesondere weil ihre wirtschaftlichen Interessen dadurch beeinflusst werden konnten. Das Röntgenprotokoll hatte jedoch nicht eine genügende Ausbreitung in der Deutschen Tierärzteschaft gefunden. Ein großer Teil, auch von spezialisierten Pferdetierärzten, kannte dieses Protokoll nicht oder setzte es aus anderen Gründen nicht ein. Wie bereits in der Vergangenheit kümmerte sich nunmehr die Gesellschaft für Pferdemedizin intensiv, insbesondere durch finanzielle Unterstützung, um die Überarbeitung des Protokolls, die am 9. Mai 2001 vom Ausschuss "Pferde" der Bundestierärztekammer empfohlen wurde. Das Ergebnis liegt nunmehr in Form des Röntgenleitfadens vor. Den Mitgliedern der 2. Röntgenkommission (Prof. Gerhards, München, Prof. Hertsch, Berlin, Dr. Jahn, Bargteheide, und Dr. von Saldern, Telgte) gebührt herzlicher Dank für die aufopferungsvolle, zeitraubende, diskussionsreiche und ehrenamtliche Arbeit an dem nun neu geschaffenen Leiffaden für die röntgenologische Beurteilung bei der Kaufuntersuchung des Pferdes (Röntgenleiffaden). Dieser Leiffaden, erweitert um die Regionen Knie und Rücken, gibt den derzeitigen Stand der Erfahrungen der Pferdepraxis wieder. Er muss neuen abgesicherten wissenschaftlichen Erkenntnissen ständig angepasst werden. Der Röntgenleiffaden wird in Verbindung mit der neuen Regelung des Kaufrechtes beim Pferd einen wertvollen Anteil für die Regelung der Aufgaben in der Zukunft tragen.
\end{abstract}

Prof. Dr. B. Hertsch

\section{Präambel}

Das Röntgenprotokoll ist ein Leitfaden für Tierärzte zur Beurteilung der gesundheitlichen Bedeutung röntgenologischer Befunde bei der Kaufuntersuchung von Pferden.

Es ist eine Empfehlung der Bundestierärztekammer, basierend auf dem Ergebnisprotokoll (1993) der ersten Röntgenkommission (Prof. Dr. Dik, Prof. Dr. Hertsch, Prof. Dr. Ueltschi), geprüft vom "Ausschuss Pferde" der Bundestierärztekammer, erarbeitet von der Gesellschaft für Pferdemedizin (GPM) - zweite Röntgenkommission (Prof. Dr. Gerhards, Prof. Dr. Hertsch, Dr. Jahn und Dr. von Saldern) und begutachtet von allen deutschen Hochschulen (Pferdekliniken) und Dr. Stihl (Schweiz).

Die röntgenologische Untersuchung umfasst Standardprojektionen, die durch ergänzende und spezielle Aufnahmen erweitert werden können. Nicht alle röntgenologischen Befunde sind mit diesen Standardprojektionen erfassbar.
Die Erweiterung oder Reduzierung der Untersuchung erfolgt nach Absprache mit dem Auftraggeber.

Die Beurteilung im Leiffaden bezieht sich auf die Standardprojektionen.

Alle darüber hinausgehenden Aufnahmen werden individuell beurteilt.

Die erhobenen röntgenologischen Befunde werden in Klassen eingeteilt.

Das Ergebnis der klinischen Untersuchung einschließlich Vorbericht, Alter, Zuchtrichtung und Nutzung kann in die Klasseneinteilung einfließen.

Eine exakte Lokalisation eines lahmheitsverursachenden Schmerzes ist im Rahmen von Kaufuntersuchungen nicht möglich.

Dieser Leitfaden gibt den derzeitigen Stand der Erfahrungen der Pferdepraxis wieder. Er muss neven abgesicherten wissenschaftlichen Erkenntnissen ständig angepasst werden. 


\section{Anmerkungen zu Qualität und Technik}

Die Geräteeinstellung muss in der Qualität Röntgenaufnahmen ergeben, mit der Knochenstrukturen, Konturen, Gelenklinien und Weichteilbereiche zu beurteilen sind.

Für die Dokumentation der Röntgenaufnahmen (Kennzeichnung und Beschriftung) dürfen nur Verfahren eingesetzt werden, die nicht nach der Entwicklung der Aufnahme auf dem Röntgenbild angebracht werden. Auf jeder Aufnahme muss die Kennzeichnung und Beschriftung lesbar sein. Die Kennzeichnung kann durch Abkürzungen für vorne rechts, vorne links, hinten rechts und hinten links erfolgen. Im Zweifelsfall gilt die Regel, dass die Kennzeichnung stets "von der Körpermitte weg” angebracht ist. Die Beschriftung sollte den Namen des Besitzers, den Namen des Pferdes, wenn bekannt die Lebensnummer, das Aufnahmedatum und den Hersteller der Röntgenaufnahme angeben.

Die Zuordnung der Röntgenaufnahme zu einem Pferd muss zweifelsfrei möglich sein.

Das Entfernen der Hufeisen wird sowohl für die Zehe $90^{\circ}$ als auch für die Oxspring-Aufnahme empfohlen. Die Entfernung der Hufeisen ist notwendig, wenn wichtige Bereiche durch das Hufeisen nicht beurteilbar sind, oder dadurch weitere Qualitätsmängel zu befürchten sind. Wird keine, wegen der Überlagerung notwendige, neue Röntgenaufnahme angefertigt, sollte eine Absprache mit den Parteien erfolgen.

Unklare, undeutliche oder verdächtige Befunde auf den Standardaufnahmen sollten durch spezielle Aufnahmen abgesichert werden.

\section{Empfehlung zur Technik der Standardaufnahmen:}

\section{Zehe $90^{\circ}$ (Übersichtsaufnahme)}

Diese Aufnahme in der Übersicht wird für die Vorder- und Hintergliedmaße als unverzichtbar angesehen.

Für diese Aufnahme können zwei unterschiedliche Techniken eingesetzt werden:

1. plane Fußung

2. auf dem Oxspringklotz

Abgebildet sein müssen die Konturen der Hufkapsel, einschließlich des Bereiches der Hufspitze (noch Zehe 90\%) (Vordergliedmaße) und proximal Fesselgelenk und Gleichbeine. Mit der Übersichtsaufnahme Zehe $90^{\circ}$ ist eine gleichzeitige orthograde Darstellung des Hufgelenkes und des Strahlbeins sowie des Fesselgelenkes und der Gleichbeine nicht möglich.

\section{Oxspring-Aufnahme}

Das Strahlbein soll in der unteren Hälfte des Kronbeins abgebildet werden. Der distale Rand des Strahlbeins darf sich nicht mit dem Hufgelenkspalt decken und die Qualität muss die Beurteilung von Kontur und Struktur des Strahlbeins erlauben. Huf- und Kronbein sollten auf dieser Übersichtsaufnahme mit abgebildet sein.

\section{Tarsus}

Für die Standarduntersuchung werden mindestens zwei Aufnahmerichtungen $\left(45^{\circ}-70^{\circ}\right.$ und $\left.90^{\circ}-115^{\circ}\right)$ benötigt. Auf diesen Aufnahmen müssen der Calcaneus und der proximale Bereich des Os metatarsale III dargestellt sein.

\section{Empfehlung zur Technik der erweiterten Röntgenuntersuchung:}

\section{Knie}

Aufnahmerichtungen: $90-115^{\circ}$ und $180^{\circ}$

Dargestellt sein müssen die Kniescheibe, der distale Anteil des Femurs und der proximale Bereich der Tibia einschließlich des Fibulakopfbereiches.

Bei der Untersuchung am stehenden Pferd muss insbesondere bei der $180^{\circ}$ Aufnahme mit einer eingeschränkten Qualität gerechnet werden.

\section{Rücken (Dornfortsätze)}

Aufnahmerichtung: $90^{\circ}$ bzw. $270^{\circ}$

Dargestellt sein müssen die dorsalen Anteile der Dornfortsätze und die dazugehörigen Zwischenräume. Am stehenden Pferd ist ihre Darstellung nur in Teilen der Brust- und vorderen Lendenwirbelsäule möglich. In der anschließenden Beurteilung werden die Wirbelgelenke nicht berücksichtigt.

\section{Befundbeschreibung}

Besondere Befunde (röntgenologisch-pathologische Befunde) können in Ausmaß und Lokalisation in die Skizzen eingezeichnet werden. Nicht eingezeichnet werden Artefakte und Befunde, die allgemein als röntgenologisch-anatomische (biologische) Varianten gedeutet werden. Zur Unterstützung der Befundbeschreibung liegen Röntgenskizzen der Standardaufnahmen von Zehe, Tarsus, Knie und Dornfortsätzen bei.

Die Befundbeschreibung mit Worten sollte umfassen:

\section{Art oder Struktur}

Aufhellung oder Verschattung, verminderte oder verstärkte Strukturdichte

\section{Form oder Kontur}

Formbeschreibung, Zubildung oder Defekt

3. Größe oder Ausmaß

z. B. Länge und Breite in Millimeter

4. Lokalisation

\section{Beurteilung}

Für die Beurteilung wird eine Einteilung in folgende vier Klassen vorgeschlagen. Diese Einteilung kommt sowohl der Fragestellung der Pferdekäufer und -verkäufer, als auch der Aussagemöglichkeit der Tierärzte entgegen:

\section{Klasse I:}

Röntgenologisch ohne besonderen Befund und Befunde, die als anatomische Formvarianten eingestuft werden

\section{Klasse II:}

Befunde, die gering von der Norm abweichen, bei denen klinische Erscheinungen unwahrscheinlich sind

\section{Klasse III:}

Befunde, die deutlich von der Norm abweichen, bei denen klinische Erscheinungen wenig wahrscheinlich sind 


\section{Klasse IV:}

Befunde, die erheblich von der Norm abweichen, bei denen klinische Erscheinungen wahrscheinlich sind (noch Beurteilung)

Die Klassifizierung der Zuordnung ist die Meinung des Interpreten.

\section{Zwischenklassen:}

Die Unterteilung in Klassen I-II, II-III und III-IV ist möglich. Sie soll zum Ausdruck bringen, dass verschiedene Untersucher möglicherweise zu unterschiedlichen Ergebnissen kämen. Eine weitere Unterteilung ist nicht vorgesehen.

Die Befunde der Klasse II können, die der Klassen III und IV müssen bei der Befundbeschreibung erwähnt werden.
Die Gesamtbeurteilung und Klassifizierung der Röntgenbefunde eines Pferdes berücksichtigt die Anzahl und die Erheblichkeit der Einzelbefunde. Sie entspricht mindestens der höchsten Einzelbeurteilung.

Eine Vielzahl von Einzelbefunden kann zu einer Einstufung in eine höhere Klasse führen.

Die röntgenologische Beurteilung kann auftragsabhängig sowohl aus alleiniger Beurteilung der Röntgenaufnahmen, als auch unter Berücksichtigung des Vorberichtes und des Ergebnisses der klinischen Untersuchung erfolgen. Nach vorangegangenen Operationen erfolgt die Beurteilung entsprechend dem aktuellen röntgenologischen Befund.

Die endgültige Entscheidung über die Zuordnung der röntgenologischen Befunde in die Klassen wird unter Berücksichtigung der klinischen Untersuchung gefällt.

\section{Zehe $90^{\circ}$}

\section{Hufwinkel}

1.1
1.2
1.3
1.4
1.5
1.6

$$
\begin{aligned}
& \text { vorne } \\
& \text { hinten } \\
& \text { vorne } \\
& \text { hinten } \\
& \text { vorne } \\
& \text { hinten }
\end{aligned}
$$
$45-55^{\circ}$
$50-55^{\circ}$
$40-45^{\circ}$ und $55-60^{\circ}$

\begin{tabular}{|c|c|c|c|c|}
\hline $\begin{array}{l}1.7 \\
1.8 \\
1.9 \\
1.10 \\
1.11 \\
1.12 \\
1.13 \\
1.14 \\
1.15 \\
1.16\end{array}$ & ungebrochen & $\begin{array}{l}\text { vorne } \\
\text { hinten } \\
\text { vorne } \\
\text { hinten } \\
\text { vorne } \\
\text { hinten } \\
\text { im Hufgelenk } \\
\text { im Krongelenk }\end{array}$ & $\begin{array}{l}45-50^{\circ} \\
50-55^{\circ} \\
40-45^{\circ} \text { und } 55-60^{\circ} \\
45-50^{\circ} \text { und } 55-60^{\circ} \\
<40^{\circ} \text { und }>60^{\circ} \\
<45^{\circ} \text { und }>60^{\circ} \\
\text { als Beugung } \\
\text { als Überstreckung } \\
\text { als Beugung } \\
\text { als Überstreckung }\end{array}$ & 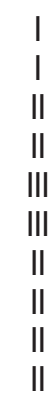 \\
\hline
\end{tabular}
$45-50^{\circ}$ und $55-60^{\circ}$
$<40^{\circ}$ und $>60^{\circ}$
$<45^{\circ}$ und $>60^{\circ}$

\section{Zehenachse: Aufnahmetechnik plane Fußung}

\section{Parallelität Hufwand - Hufbein}

1.17

1.18

parallel

1.19

nicht parallel

\section{Abstand Hufwand - Hufbein (Warmblüter)}

gemessen im rechten Winkel von der Mitte des Hufbeines
1.20
1.21
$<1,5 \mathrm{~cm}-1,8 \mathrm{~cm}$
1.22

\section{Hufbein - Margo solearis}

glatt in der dorsalen Hälfte
1.23
leicht unregelmäßig konturiert in der palmaren/plantaren Hälfte
1.24
Abbruch, Fraktur einschließlich "Hufbeinspitze"
1.25
Zubildung an der Kontur
1.26
starke unregelmäßige Kontur der hinteren Hälfte bis zur Osteolyse 


\section{Dorsale Hufbeinwand}

1.29

1.30

gestreckter Verlauf mit glatter Kontur

I

1.31

krallenförmige Deformierung

II

1.32

Aufbiegung der Hufbeinspitze

II bis III

Zubildung in der unteren Hälfte, glatt konturiert

II

1.33

Zubildung unregelmäßig konturiert

III

\section{Processus extensorius}

rund, schmal oder breit, eckig oder kantig

$1.34 \quad$ (kein Randwulst), zweigipfelige Kontur glatt

1.35

spitz ausgezogener Randwulst

1.36

1.37

mehrfach spitzzackig

kleine isolierte Verschattung ohne einen entsprechenden Defekt

oder knöcherne Zubildungen im dorsalen Bereich des Hufoder Kronbeines II

1.38

isolierte Verschattung mit unterschiedlicher Deutung

III

Fraktur an der Basis

IV

\section{Hufbeinäste $90^{\circ}$}

1.40 Authellungslinien (Deutung als Fissur oder Fraktur)

1.41 isolierte Verschattung am Hufbeinast

IV

II

\section{Strahlbein $90^{\circ}$}

1.42

1.43

1.44

1.45

1.46

1.47

1.48

1.49

1.50
Sklerosierung der Spongiosa (Zehe $90^{\circ}$ mit Raster)

Osteoporose der Spongiosa

Zubildung (oder isolierte Verschattung) am Strahlbeinseitenende oder im Bereich des proximalen Randes

zentrale Delle (flache Konkavität) des Sagittalkammes

Usur, zentraler Einbruch (scharf begrenzter Defekt) zystoider Defekt

Fragmente im Strahlbein-Hufbein-Band

Randexostosen Strahlbein - Hufbein Hilfsgelenkfläche

Diskrepanz im Vergleich der Befunde rechts - links
III bis IV

II bis III

II bis III

IV

IV

II bis III

II bis III

II bis III

\section{Hufknorpelverknöcherung $90^{\circ}$}

1.51

1.52

1.53

1.54 am Ansatz (ein- oder beidseitig)

vollständig

isoliert im Hufknorpel

Aufhellungslinie im verknöcherten Hufknorpel
II

II bis III

II

III

\section{Hufgelenk $90^{\circ}$}

1.55

1.56

1.57

1.58

1.59

1.60
Gelenkspalt gleichmäßig

Konturveränderung an der Huf- oder Kronbeinfläche

Strukturveränderungen im subchondralen Gelenkflächenbereich

Zubildungen Kronbein Randwulst dorsal

Zubildungen Kronbein Randwulst palmar und/oder Strahlbein Margo proximalis

Zubildungen dorsal auf der Kronbeinkontur, unregelmäßig, rauh
I

III bis IV

III bis IV

II bis III

III bis IV

III bis IV

\section{Kronbein $90^{\circ}$}

1.61

1.62

1.63

1.64
Dorsale Zubildungen (proximal, extraartikulär)

Zubildung an der Kronbeinlehne, distal gerichtet zystoider Defekt

Zubildung palmar/plantar am Ansatz der oberflächlichen Beugesehne
II bis III

II

III bis IV

II bis III

\section{Krongelenk $90^{\circ}$}

1.65

Subluxation

Zubildungen

1.66
Randexostose dorso-proximal

klein, glatt
III

II bis III 


\section{Fesselbein $90^{\circ}$}

1.72

1.73

1.74

1.75

1.76

1.77

1.78
Zubildungen palmar / plantar (Leist) Zubildung

\section{Fesselgelenk $90^{\circ}$}

1.79

1.80

1.81

1.82

1.83

1.84

1.85

1.86

1.87
Zubildung distodorsal Mc III / Mt III

Zubildung Randexostose dorsoprox. Fesselbein

Mc III / Mt III Randexostose suprakondylär

Einkerbung mit oder ohne Fragmente auf dem Sagittalkamm dorsal (Osteochondrosis) vergrößerter periartikulärer Weichteilschatten isolierte Verschattung im dorsalen Bereich isolierte Verschattung palmar/plantar kleiner als Pfefferkorn größer als Pfefferkorn suprakondyläre Deformation - Einbiegung (palmar oder dorsal)
II bis III

$$
\text { II }
$$

III

III bis IV

III bis IV

III bis IV

III bis IV

\section{Gleichbeine $90^{\circ}$}

1.88

1.89

1.90

1.91

1.92

1.93

1.94

1.95

1.96

1.97

1.98

1.99

1.100

1.101

1.102
Randexostosen Facies articularis grobmaschige Struktur Osteolyse oder zystoider Defekt Zubildungen (Fesselringband)

Zubildung Apex

Zubildung Basis schmale Gefäßkanalzeichnung Strukturauflösungen im Bereich der Gefäßkanäle vorne hinten isolierte Verschattung proximal des Gleichbeins kleiner als Pfefferkorn größer als Pfefferkorn

Aufhellungslinie Fissur/Fraktur deutlicher Größenunterschied der Gleichbeine im Vergleich lateral - medial (projektionsbedingte Vergrößerung berücksichtigen) glatte Kontur und gleichmäßige Struktur unregelmäßige Kontur und ungleichmäßige Struktur vergrößerter Abstand zwischen Fesselbein und Gleichbein
II bis III

II bis III

II bis III

III

III

II bis III

II bis III III

III
II bis III II bis III III bis IV II bis III II bis III II II III II bis III II bis III III bis IV I

II bis III III bis IV II bis III

\section{Oxspring-Aufnahme $0^{\circ}$}

\section{Strahlbein (Oxspring)}

Deutliche Diskrepanz im Vergleich der Befunde rechts - links

bezüglich Größe,

und/oder Form,

und/oder Form und Anzahl der Canales 
Länge der Canales

distal - schräger Seitenteil

Form der Canales

über $1 / 4$ der Strahlbeinbreite

schmal, spitz, breit,

konisch oder zylindrisch

kleinkolbig (bis Senfkorngröße)

großkolbig (Pfefferkorngröße und mehr)

verzweigt

Struktur

grobmaschig total

sklerosiert oder osteoporotisch

zentrale Aufhellung (zystoider Defekt)

zentrale Aufhellung (Einbruch)

grobmaschig partiell

Aufhellungslinien, Frakturverdacht

2.16

2.17

Kontur - Zubildungen

(Artefakte ausschließen!)

Seitenenden spitz

proximal

distal am Übergang zum schrägen

2.19

Seitenteil einschließlich Fragmenten

I
III bis IV
II
I bis II
II bis III
III bis IV
III bis IV
II bis III
II bis III
IV
IV
III
IV
III
III
II bis III

\section{Hufbein $0^{\circ}$}

2.20

2.21

2.22

2.23

2.24

2.25
Aufhellungslinien, Frakturverdacht (Artefakte ausschließen!)

Aufhellung (zystoider Defekt)

Hufknorpelverknöcherung (s. Zehe 90 1.51-1.54)

Kontur Margo solearis gleichmäßig oder zentral mit flacher Einziehung (Crena) großkonische oder rundliche Einziehung sehr unregelmäßige Kontur mit oder ohne isolierte Verschattung
IV

IV

II bis III

II

II bis III

III bis IV

\section{Kronbein $0^{\circ}$}

2.26

2.27

2.28
Einziehung in der Mitte der distalen Gelenkfläche

Aufhellung (zystoider Defekt)

Aufhellung (erweiterter Markraum)
II

IV

II bis III

II bis III

II bis III

II bis III

IV

III bis IV

\section{Tarsus $45^{\circ}-70^{\circ}$ und $90^{\circ}-115^{\circ}$}

\section{Talokruralgelenk}

Abflachung oder Einkerbung im Bereich des Sagittalkammes 
3.7 distal gerichtete Zubildung oder isolierte Verschattung am Talus

II bis III

freie isolierte Verschattung auch distal im Rezessus des Talokruralgelenkes

II bis III

zystoide Defekte Talus, Tibia

3.10

Zubildung proximal und distal Sustentaculum tali

III bis IV

Gelenkspalt Talus - Calcaneus

3.11 Sklerosierung und lokalisierte Aufhellungen

III bis IV

3.12

Calcaneus Osteolyse, lokale Aufhellung

IV

zystoider Defekt

IV

persistierende distale Fibulaepiphysenfuge

III bis IV

3.14

persistierende distale Tibiaepiphysenfuge

| bis ||

II

\section{Intertarsalgelenke und Tarsometatarsalgelenk}

klar durchgehende Gelenkspalten, gleichmäßige Knochenstruktur,

3.16

3.17

3.18

3.19

3.20

3.21

3.22

3.23

3.24

3.25

3.26

3.27

3.28

3.29

3.30

3.31

3.32 deutliche Synovialgruben, keine Deformierung der Tarsalknochen

verschwommene oder schmale Gelenkspalten deutlich verschmälerte Gelenkspalten (strichförmig)

Randwülste und -zacken

Randexostose am Mt III (Osteophyten)

Strukturauflösung und Usuren in den Gelenkspalten

bis $2 \mathrm{~mm}$ (Osteophyten) über $2 \mathrm{~mm}$

zystoide Defekte

Durchbauung der Gelenkspalten u. Synovialgruben

periostale Reaktionen, Kapselverknöcherung

innere Bandverknöcherung ohne Ankylosenbildung oder mit Ankylosenbildung

periostale u. desmale Reaktionen am Mt III - Mt II - Mt IV (Überbein sofern abgebildet)

sagittale Aufhellungslinie in der proximalen $\mathrm{Mt}$ III-Gelenkfläche (0-Aufnahme)

unregelmäßige Sklerosierung proximal Mt III (Insertionsdesmopathie $M$. interosseus med.)

Hypertrophie des lateralen Griffelbeinkopfes oder Os † IV (Hasenhacke)

Periostitis ossificans laterales Griffelbein (Rehbein)

Deformierung des Os tc oder Os † III
I

II

III bis IV

II bis III

III

II bis III

IV

III bis IV

III bis IV

III bis IV

IV

II bis III

III

III

III

III

III

\section{Knie $90^{\circ}-115^{\circ}$}

\section{Patella}

Vorderfläche

4.1

Konturveränderungen

4.2

Patellabasis

rund, kleiner $5 \mathrm{~mm}$

II

spitzzackig

Zubildungen größer 2 mm

4.3

4.4

4.5

4.6

4.7

Facies articularis

isolierte Verschattungen

randständig

gelenkflächennah

I

III

III

an der Facies articularis proximalis, größer als $3 \mathrm{~mm}$

III bis IV

Konturveränderungen zentral

IV

Aufhellung (zystoider Defekt)

IV

Patellaspitze

Zubildungen

4.9

4.10

4.11

4.12

isolierte Verschattung

rund, kleiner $5 \mathrm{~mm}$

spitzzackig, größer $3 \mathrm{~mm}$

$\|$

III

III

Authellungslinien (Fissur-, Frakturverdacht)

III bis IV

4.13 Aufhellung (zystoider Defekt)

IV

\section{Trochlea femoris}

4.14

4.15

Einkerbung, kranio-proximal oder distal der medialen Trochlea am Femur

4.16

Abflachung der Kontur im mittleren Drittel der lateralen Trochlea, glatt

II bis III unregelmäßige Abflachung der gesamten Trochleakontur 


\section{Femorotibialgelenk}

Eminentia intercondylaris

\section{Tuberositas tibiae}
4.27
glatte Konturveränderung
II
4.28
spitzzackige Konturveränderung (größer als $2 \mathrm{~mm}$ )
III

\section{Knie $180^{\circ}$}

4.29

medialer/lateraler Femurkondylus

Konturveränderung

II bis III

Aufhellung (zystoider Defekt)

klein (Hirse) II / mittel (Pfefferkorn) III / groß (Haselnuss) IV

II bis IV

Zubildung medialer Tibiakondylus (Insertion)

Fibula eine oder mehrere querverlaufende Aufhellungslinien

Zubildungen zwischen Fibula und Tibia (adultes Pferd)

Authellungslinie Basis Eminentia intercondylaris

Aufhellung im Tibiakopf (zystoider Defekt)

lateral/medial

Area intercondylaris caudalis

\section{Rücken}

\section{Dornfortsätze}

\section{1 \\ Widerrist}

Frakturen

Bereich Sattellage und Lendenwirbelsäule

Gesellschaft für Pferdemedizin e. V.

Posffach 5502 51, D-44210 Dortmund Telefon/Telefax 0231-737399

http://www.g-p-m.org, info@g-p-m.org
Bundestierärztekammer e. V. Oxfordstraße 10, D-531 11 Bonn

Telefon: 0228-725460, Telefax: 7254666

http://www.vetline.de/btk, geschaeftsstelle@btk-bonn.de 
Röntgenskizzen zu den Standardaufnahmen der Zehe

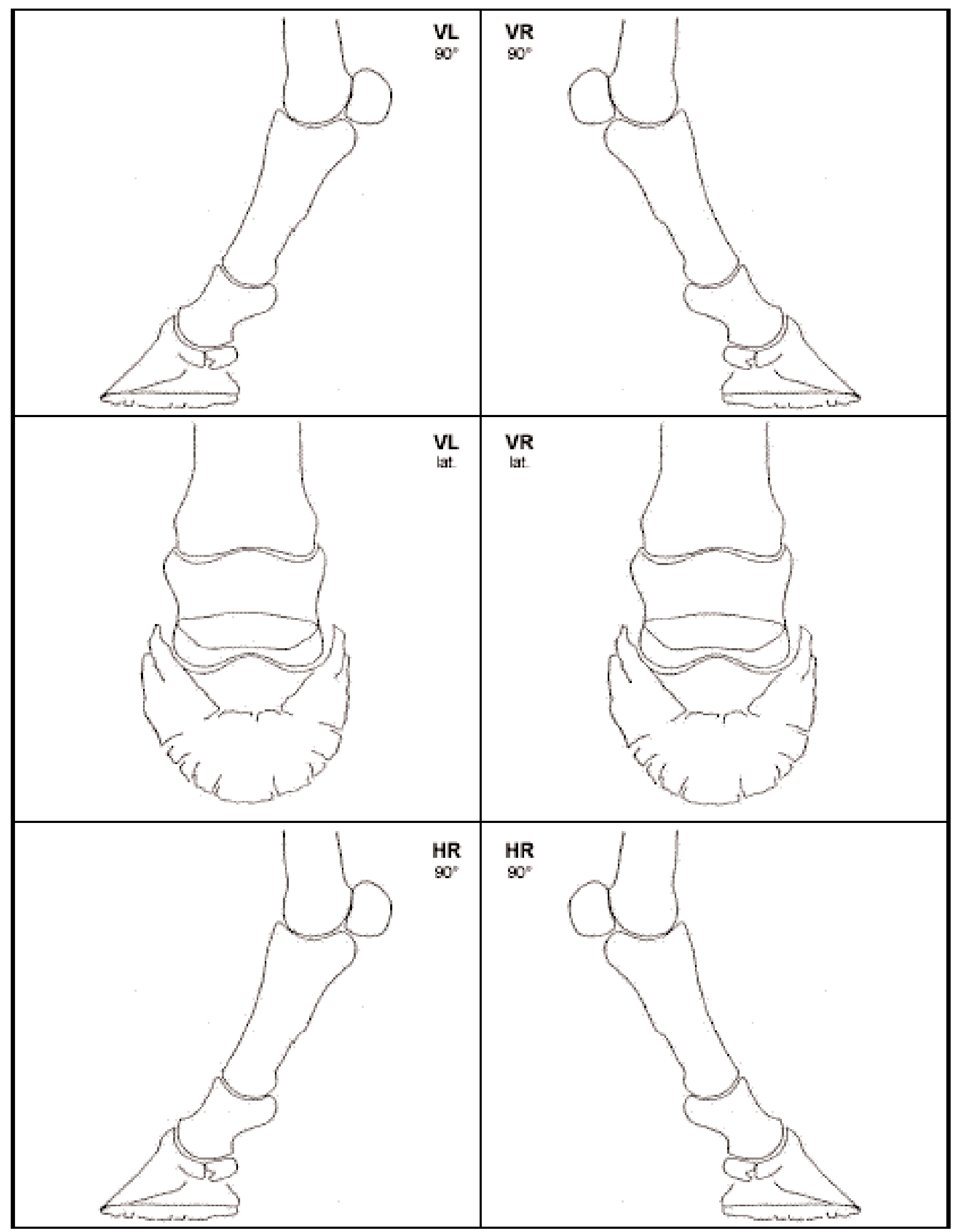


Röntgenskizzen zu den Standardaufnahmen des Tarsus

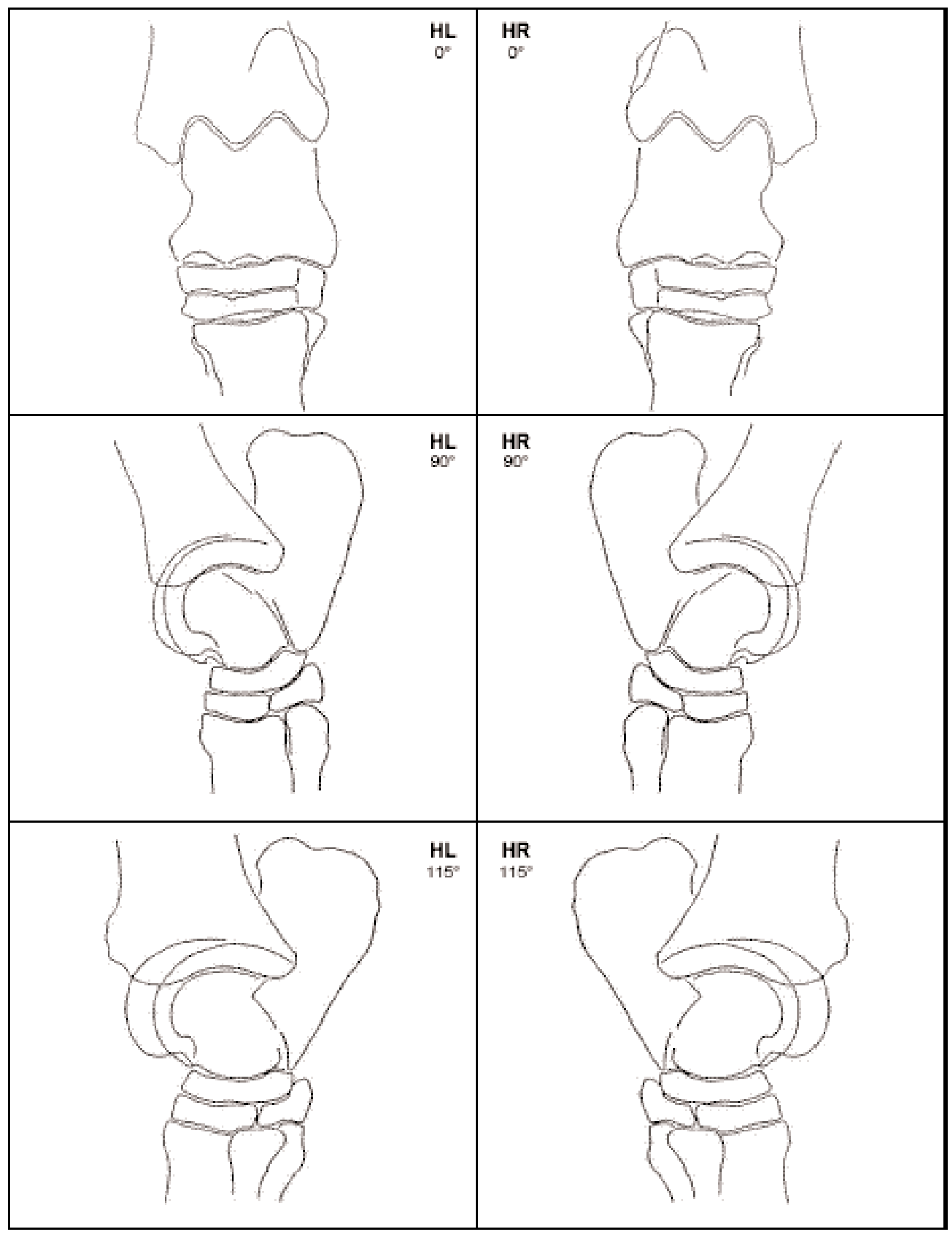


Autoren

Röntgenskizzen zu den Standardaufnahmen des Knies

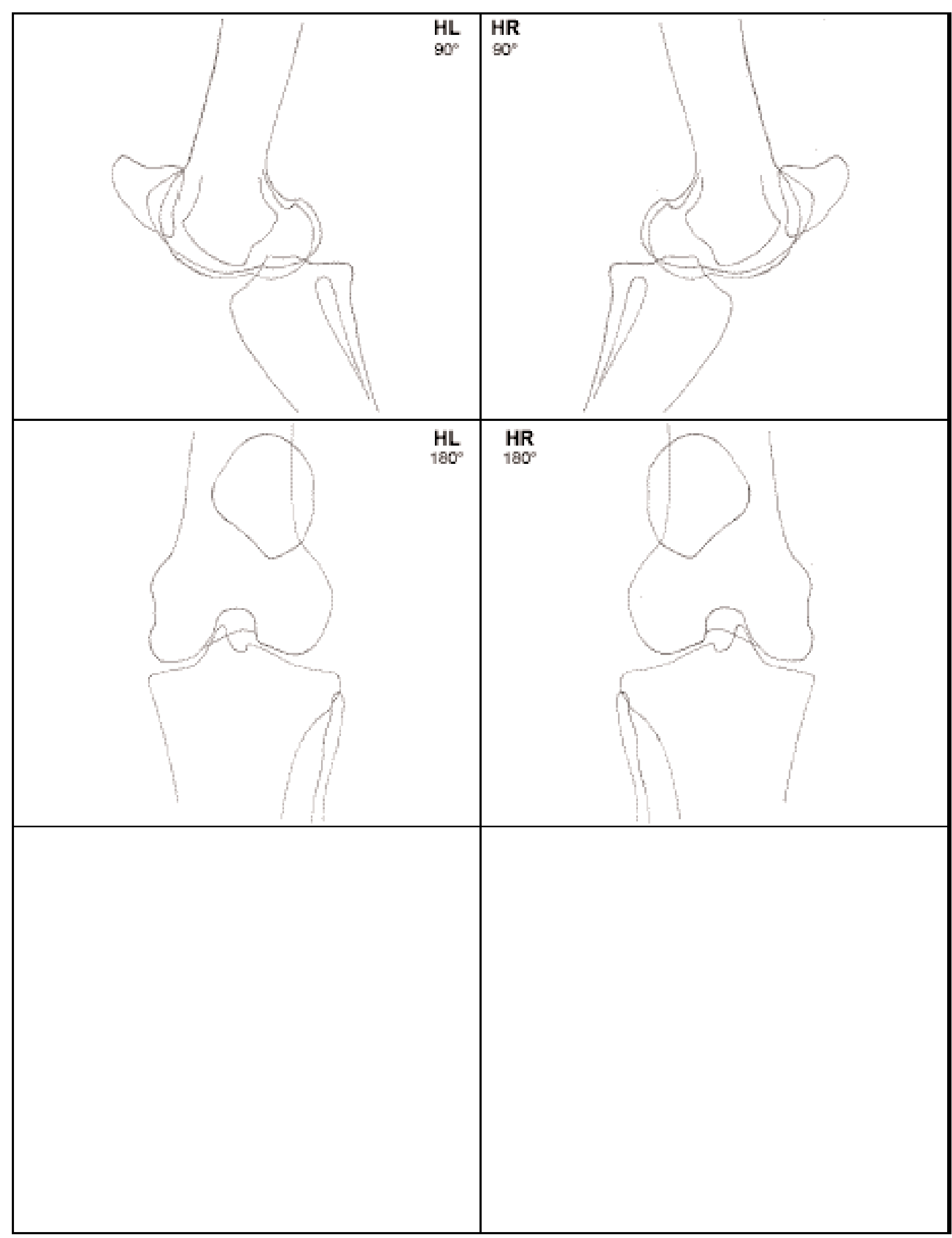

Pferdeheilkunde 19

197 
Röntgenskizzen zu den Standardaufnahmen des Rückens

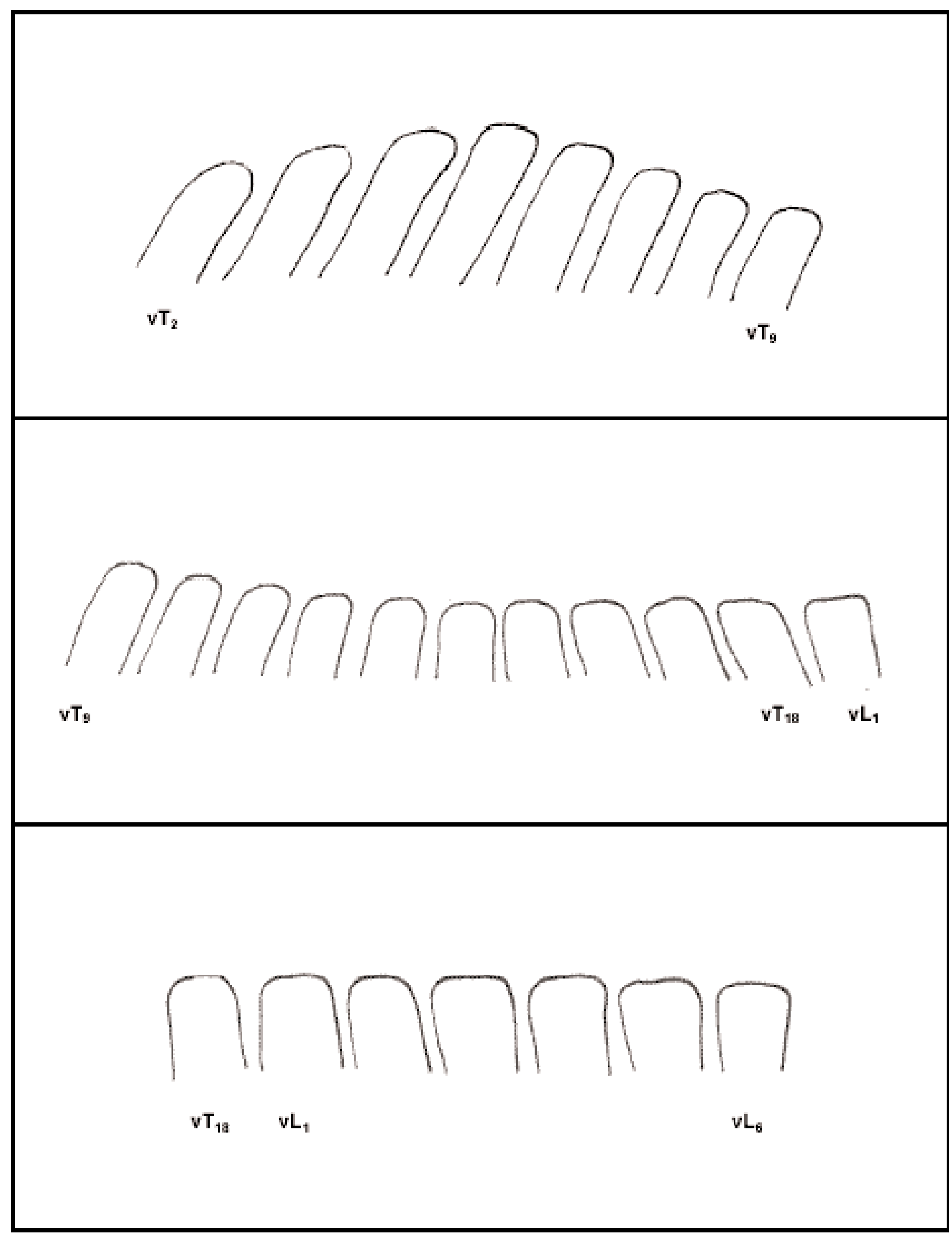

